\title{
Novel synthesis of benzimidazole by Ring Contraction Rearrangement of benzodiazepine
}

\author{
A. TIMOTOU, A. ADJOU*, M.V. SAY, D. DRISSA, S.A. TOURÉ, G.C. TÉA and \\ Y.T. N'GUESSAN
}

\author{
Laboratoire de Chimie Organique Structurale - UFR SSMT - Université Félix Houphouët Boigny, \\ 22 B.P. 582 Abidjan 22 - République de Côte d'Ivoire. \\ *Corresponding author, E-mail: adjouane@hotmail.com
}

\begin{abstract}
Condensation of substituted aromatic ketones (acetophenone) and substituted aldehydes give unsaturated ketones 14 (chalcones) which react with $o$-phenylenediamine $\mathbf{7}$ to afford the corresponding benzodiazepines 15. Treatments of benzodiazepines under basic conditions give benzimidazole derivatives. Structures of all synthesized compounds have been characterized by their NMR and mass spectral data. (c) 2013 International Formulae Group. All rights reserved.
\end{abstract}

Keywords: $N$ '-Thioacylamidines, Chalcone, $o$-phenylenediamine, benzodiazepine, benzimidazole.

\section{INTRODUCTION}

The antihelmintic drugs derived from benzimidazole are the largest chemical family used to treat endoparasitic diseases in domestic animals and humans, characterized by their high therapeutical index in the different helmintosis, polyvalent effect, and low toxicity. Specifically, it has been estimated that 10 million people worldwide could be infected, and in the past 10 years an increase of the infection has been reported among domestic animals (pigs, horses), and wildlife, with a consequent increase among humans.

In recent years, much attention has been given to the substituents at the 1- and 2position of the benzimidazole ring which gave good antiviral activities. Many reports have revealed that the influence of the substitution at the 1,2 and 5-positions of the benzimidazole ring was very important for their pharmacological effects (Ashnagar et al., 2009; Patel and Singh, 2009). It is well known that several 1,2-disubstituted benzimidazoles 1 (DeLong, 1984; Miller et al., 1985), 2 (Porcari et al., 1998), 3 (Victor et al., 1997), 4 (Garuti et al., 1998), 5 (Swayze et al., 1993), 6 (Gardiner et al., 1995) were potent inhibitors of RNA viruses. Thus, this let us to prepare and evaluate other derivatives (Figure 1). Benzimidazole derivatives have been intensively used as drugs in medicinal chemistry such as antihelmentic (Hazelton et al., 1995), antihistaminic (Al-Muhaimeed, 1997) and antiulcerative (Richter, 1997), Facing the resistance phenomena, the development of new molecules that inhibited resistant strains revived interest in the search for novel bioactive compounds. Therefore we decided to develop novel structural analogs of benzimidazole. We expanded our research in the substitution at the 2-position. The choice 
<smiles>CC(C)[S+](=O)(=O)n1c(N)nc2ccc(/C(=N/O)c3ccccc3)cc21</smiles><smiles>Nc1nc2cc(Cl)c(Cl)cc2n1Cc1ccccc1</smiles><smiles>O=[N+]([O-])c1ccc(S(=O)(=O)n2c(CCc3ccccc3)nc3ccccc32)cc1</smiles><smiles>CC(Cn1c(=S)[nH]c2ccccc21)NCc1ccccc1</smiles>

5<smiles>C#C/C=C(\c1ccccc1)c1ccc2nc(N)n([S+](=O)[O-])c2c1</smiles>

3<smiles>CCCOn1c(CC)nc2ccc(Cl)cc21</smiles>

Figure 1: 1,2-disubstituted benzimidazoles.

of chains linked at the $\mathrm{C}-2$ was made in view of their presence in other classes of antiviral compounds. In this paper, we describe the synthesis of benzimidazole. As strategy approach, we used 1,5-benzodiazepines as intermediaries which were converted to benzimidazole under basic conditions.

\section{MATERIALS AND METHODS}

\section{General}

Melting points were determined with a Kofler hot-stage apparatus and are uncorrected. ${ }^{1} \mathrm{H}$ NMR and ${ }^{13} \mathrm{C}$ NMR were recorded on a Bruker Avance $300 \mathrm{MHz}$ spectrometer instrument using TMS as an internal standard. Mass spectrometric measurements were performed using HP5989X instrument. Column chromatography was carried out over silica gel 60 (0.04-0.06 mm) (Merck AG Darmstadt, Germany). All spectrometers analysis were realized at laboratory CEISAM of Nantes University.

\section{General method to synthesis of benzodiazepine}

To a solution of chalcone 14 (35 mmol) in ethanol $(30 \mathrm{~mL})$, a few drops of triethylamine and $o$-phenylenediamine (un or substituted) $(42 \mathrm{mmol})$ were added. The mixture was heated under reflux for 10 hours in the dark. The mixture was cooled at room temperature and then put in the freezer overnight. The solid product $\mathbf{1 5}$ was separated, filtered, and washed with cold ethanol. The residue was purified by column chromatography on silica gel.

\section{4-diphenyl-2,3-dihydro-1,5-benzodiazepine}

\section{$15 \mathbf{a}$}

From 1,3-diphenylpropenone $\quad \mathbf{1 4 a}$ (7.29 g, $35 \mathrm{mmol}$ ) and o-phenylenediamine 7a (4.54 g, $42 \mathrm{mmol})$ was obtained 15a (5.32 g; $51 \%)$ as yellow crystals; $\mathrm{R}_{\mathrm{f}}$ : 0.4 (hexane/dichloromethane v/v: 70/30); mp: $155-156{ }^{\circ} \mathrm{C}$.

${ }^{1} \mathrm{H} \mathrm{NMR}\left(\mathrm{CDCl}_{3}, 300 \mathrm{MHz}\right) \delta: 3.05-$ $3.08\left(\mathrm{~m}, 2 \mathrm{H}, \mathrm{CH}_{2}\right) ; 5.22(\mathrm{~d}, 1 \mathrm{H}, \mathrm{NH}) ; 5.87-$ $5.88\left(\mathrm{~m}, 1 \mathrm{H}, \mathrm{H}_{2}\right)$; 6.80-7.80 $(\mathrm{m}, 14 \mathrm{H}$, aromatic protons).

${ }^{13} \mathrm{C}$ NMR $\left(\mathrm{CDCl}_{3}, 75 \mathrm{MHz}\right) \delta: 37.7$ $\left(\mathrm{C}_{3}\right)$; $64.4\left(\mathrm{C}_{2}\right) ; 118.3\left(\mathrm{C}_{9}\right) ; 121.7\left(\mathrm{C}_{7}\right) ; 125.3$ $\left(\mathrm{C}_{6}\right) ; 128.6\left(\mathrm{C}_{17}, \mathrm{C}_{21}\right) ; 131.1\left(\mathrm{C}_{8}\right) ; 131.9\left(\mathrm{C}_{18}\right.$, $\left.\mathrm{C}_{20}\right) ; 132.2\left(\mathrm{C}_{19}\right) ; 132.3\left(\mathrm{C}_{12}, \mathrm{C}_{14}\right) ; 133.1\left(\mathrm{C}_{11}\right.$, $\left.\mathrm{C}_{15}\right)$; $133.8\left(\mathrm{C}_{13}\right) ; 135.5\left(\mathrm{C}_{10}\right) ; 138.8\left(\mathrm{C}_{5 \mathrm{a}}\right)$; $143.5\left(\mathrm{C}_{16}\right) ; 146.6\left(\mathrm{C}_{9 \mathrm{a}}\right) ; 165.8\left(\mathrm{C}_{4}\right)$.

Mass $(\mathrm{m} / \mathrm{z})=\mathrm{M}^{+}=298(26) ; \mathrm{m} / \mathrm{z}(\%)$ $=221.25$ (25); 194.20 (100); 91(21.26); 77 (20.25); 63 (12.25). 
(4-methoxyphenyl)-4-(o-hydroxyphenyl)-2,3dihydroxy-1,5-benzodiazepine $15 b$

From 1-(o-hydroxyphenyl)-3-(4methoxyphenyl)propenone 14b (8.90 g, 35 mmol) and o-phenylenediamine 7a (4.54 g, 42 mmol) was obtained $\mathbf{1 5 b}(6.74 \mathrm{~g} ; 56 \%)$ as yellow crystals; $\mathrm{R}_{\mathrm{f}}: 0.5$ (hexane/ethyl acetate v/v : 80/20); mp: $140-141^{\circ} \mathrm{C}$.

${ }^{1} \mathrm{H}$ NMR $\left(\mathrm{CDCl}_{3}, 300 \mathrm{MHz}\right)$ \%: 3.04 (dd, $1 \mathrm{H}, \mathrm{H}_{3 \alpha}, \mathrm{J}=9 \mathrm{~Hz}$, and $15 \mathrm{~Hz}$ ), 3.32 (dd, $1 \mathrm{H}, \mathrm{H}_{3 \beta}, \mathrm{J}=6 \mathrm{~Hz}$, and $\left.15 \mathrm{~Hz}\right), 3.83(\mathrm{~s}, 4 \mathrm{H}$, $\mathrm{OCH}_{3}$ et $\left.\mathrm{NH}\right), 5.15\left(\mathrm{dd}, 1 \mathrm{H}, \mathrm{H}_{2}, \mathrm{~J}=6 \mathrm{~Hz}\right.$ and $9 \mathrm{~Hz})$; 6.76-7.37 (m, 12H, aromatic protons); 15.30 (s, $1 \mathrm{H}, \mathrm{OH}),{ }^{13} \mathrm{C} \mathrm{NMR}\left(\mathrm{CDCl}_{3}, 75\right.$ $\mathrm{MHz})$ \&: $36.7\left(\mathrm{CH}_{3}\right)$; $55.4\left(\mathrm{C}_{2}\right) ; 69.3\left(\mathrm{C}_{3}\right)$; $114.3\left(\mathrm{C}_{18}, \mathrm{C}_{20}\right) ; 114.8\left(\mathrm{C}_{9}\right) ; 116.2\left(\mathrm{C}_{12}\right)$; $120.4 \quad\left(\mathrm{C}_{7}\right) ; 120.8 \quad\left(\mathrm{C}_{10}\right) ; 124.2 \quad\left(\mathrm{C}_{14}\right)$; 127.1( $\left.\mathrm{C}_{6}\right) ; 132.7\left(\mathrm{C}_{17}, \mathrm{C}_{21}\right) ; 133.6\left(\mathrm{C}_{8}\right) ; 135.7$ $\left(\mathrm{C}_{15}\right) ; \quad 136.5 \quad\left(\mathrm{C}_{13}\right) ; \quad 136.8 \quad\left(\mathrm{C}_{16}\right) ; 137.1$ $\left(\mathrm{C}_{5 \mathrm{a}}\right) ; 145.0\left(\mathrm{C}_{9 \mathrm{a}}\right) ; 150.5\left(\mathrm{C}_{19}\right) ; 162.0\left(\mathrm{C}_{11}\right)$; $171.2\left(\mathrm{C}_{4}\right)$. Mass- $(\mathrm{m} / \mathrm{z})=344 . \mathrm{M}^{+}=344 ; \mathrm{m} / \mathrm{z}$ (\%); M+ 2 = 346 (16.41); M+1 = 345 (72.32); 240 (100); 119 (32.96); 91 (35.85) 77 (11.40).

\section{Thienyl-4-tolyl-2,3-dihydro-1,5-}

benzodiazepine 15 c

From 1-tolyl-3-thienyl propenone 14c (7.99 g, $35 \mathrm{mmol}$ ) and o-phenylenediamine 7a (4.54 g, $42 \mathrm{mmol})$ was obtained $15 \mathrm{c}$ (5.01 g; $45 \%$ ) as yellow crystals; $\mathrm{R}_{\mathrm{f}}$ : 0.4 (hexane/ethyl acetate v/v: 80/20); $\mathrm{mp}: 125-126^{\circ} \mathrm{C}$.

${ }^{1} \mathrm{H}$ NMR $\left(\mathrm{CDCl}_{3}, 300 \mathrm{MHz}\right) \delta: 2.38(\mathrm{~s}$, $\left.1 \mathrm{H}, \mathrm{CH}_{3}\right) ; 2,99\left(\mathrm{dd}, 1 \mathrm{H}, \mathrm{H}_{\mathrm{A}},{ }^{3} \mathrm{~J}_{\mathrm{AX}}=9 \mathrm{~Hz},{ }^{2} \mathrm{~J}_{\mathrm{AM}}\right.$ $=13.2 \mathrm{~Hz}) ; 3.26\left(\mathrm{dd}, 1 \mathrm{H}, \mathrm{H}_{\mathrm{M}},{ }^{3} \mathrm{~J}_{\mathrm{BX}}=4.2 \mathrm{~Hz}\right.$, $\left.{ }^{2} \mathrm{~J}_{\mathrm{AM}}=13.2 \mathrm{~Hz}\right) ; 3,72(\mathrm{~m}, 1 \mathrm{H}, \mathrm{NH}) ; 5.50(\mathrm{dd}$, $\left.1 \mathrm{H}, \mathrm{H}_{\mathrm{X}},{ }^{3} \mathrm{~J}_{\mathrm{AX}}=9 \mathrm{~Hz},{ }^{2} \mathrm{~J}_{\mathrm{MX}}=4.2 \mathrm{~Hz}\right) ; 6.78$ $7.78(\mathrm{~m}, 11 \mathrm{H}$, aromatic and thienyl protons).

${ }^{13} \mathrm{C} \mathrm{NMR}\left(\mathrm{CDCl}_{3}, 75 \mathrm{MHz}\right) \delta: 21.4$ $\left(\mathrm{CH}_{3}\right) ; 37.7\left(\mathrm{C}_{3}\right) ; 66.8\left(\mathrm{C}_{2}\right) ; 121.5\left(\mathrm{C}_{9}\right) ; 125.4$ $\left(\mathrm{C}_{7}\right) ; 128.7\left(\mathrm{C}_{6}\right) ; 128.9\left(\mathrm{C}_{18}\right) ; 132.1\left(\mathrm{C}_{20}\right)$; $133.4\left(\mathrm{C}_{16}\right) ; 134.0\left(\mathrm{C}_{8}\right) ; 134.9\left(\mathrm{C}_{19}\right) ; 135.2$ $\left(\mathrm{C}_{11}, \mathrm{C}_{15}\right) ; 136.2\left(\mathrm{C}_{12}, \mathrm{C}_{14}\right) ; 138.2\left(\mathrm{C}_{10}\right) ; 140.5$ $\left(\mathrm{C}_{5 \mathrm{a}}\right) ; 142.6\left(\mathrm{C}_{13}\right) ; 148.4\left(\mathrm{C}_{9 \mathrm{a}}\right) ; 167.2\left(\mathrm{C}_{4}\right)$.

Mass $(\mathrm{m} / \mathrm{z})=318 . \mathrm{M}^{+}=318(10) ; \mathrm{m} / \mathrm{z}(\%)$; 319 (5); 208 (100); 110 (20.15); 91 (21.26); 77 (11.91); 63 (12.25); 39 (25,1).

Phenyl-2-Thienyl-2,3-dihydro-1,5-

benzodiazepine 15d

From 1-phenyl-3-thienyl propenone 14d $(7.50 \mathrm{~g}, \quad 35 \mathrm{mmol})$ and ophenylenediamine $7 \mathbf{a}(4.54 \mathrm{~g}, 42 \mathrm{mmol})$ was obtained 15d (5.53 g; 52\%) as blackish crystals; $\mathrm{R}_{\mathrm{f}}: 0.4$ (hexane/ethyl acetate $\mathrm{v} / \mathrm{v}$ : 95/5); mp: $120-121{ }^{\circ} \mathrm{C}$.

${ }^{1} \mathrm{H}$ NMR $\left(\mathrm{CDCl}_{3}, 300 \mathrm{MHz}\right), \delta: 3.06$ $\left(\mathrm{dd}, 1 \mathrm{H}, \mathrm{H}_{\mathrm{A}},{ }^{3} \mathrm{~J}_{\mathrm{AX}}=9 \mathrm{~Hz},{ }^{2} \mathrm{~J}_{\mathrm{AM}}=12 \mathrm{~Hz}\right) ; 3.32$ $\left(\mathrm{dd}, 1 \mathrm{H}, \mathrm{H}_{\mathrm{M}},{ }^{3} \mathrm{~J}_{\mathrm{MX}}=6 \mathrm{~Hz},{ }^{2} \mathbf{J}_{\mathrm{AM}}=12 \mathrm{~Hz}\right) ; 3.79$ (s, $1 \mathrm{H}, \mathrm{NH}): 5.55\left(\mathrm{dd}, 1 \mathrm{H}, \mathrm{H}_{\mathrm{X}},{ }^{3} \mathrm{~J}_{\mathrm{AX}}=9 \mathrm{~Hz}\right.$, $\left.{ }^{2} \mathrm{~J}_{\mathrm{MX}}=6 \mathrm{~Hz}\right) ; 6.83-7.92(\mathrm{~m}, 12 \mathrm{H}$, aromatic and thienyl protons).

${ }^{13} \mathrm{C} \mathrm{NMR}\left(\mathrm{CDCl}_{3}, 75 \mathrm{MHz}\right) \delta: 37.9$ $\left(\mathrm{C}_{3}\right) ; 66.7\left(\mathrm{C}_{2}\right) ; 121.5\left(\mathrm{C}_{9}\right) ; 124.2\left(\mathrm{C}_{7}\right) ; 128.7$ $\left(\mathrm{C}_{6}\right) ; 128.9\left(\mathrm{C}_{18}\right) ; 131.2\left(\mathrm{C}_{20}\right) ; 132.2\left(\mathrm{C}_{16}\right)$; $134.5\left(\mathrm{C}_{8}\right) ; 134.9\left(\mathrm{C}_{19}\right) ; 136.8\left(\mathrm{C}_{11}, \mathrm{C}_{15}\right)$; $137.2\left(\mathrm{C}_{12}, \mathrm{C}_{14}\right) ; 139.2\left(\mathrm{C}_{10}\right) ; 141.5\left(\mathrm{C}_{5 \mathrm{a}}\right)$; $142.2\left(\mathrm{C}_{13}\right) ; 148.4\left(\mathrm{C}_{9 \mathrm{a}}\right) ; 167.2\left(\mathrm{C}_{4}\right)$. Mass $(\mathrm{m} / \mathrm{z})=304.4 . \mathrm{M}^{+}=304 ; \mathrm{m} / \mathrm{z}(\%): 306(5) ;$ 305 (15); 304 (75.76); 77 (100); 52 (48.44); 39 (67.26).

\section{(o-chlorophenyl)-4-phenyl-2,3-dihydroxy- 1,5-benzodiazepine 15e}

From

1-phenyl-3-(ochlorophenyl)propenone $\mathbf{1 4 e} \quad(8.49 \mathrm{~g}, \quad 35$ mmol) and o-phenylenediamine 7a (4.54 g, 42 mmol) was obtained 15e (8.81 g; 75\%) as yellow crystals; $\mathrm{R}_{\mathrm{f}}: 0.5$ (hexane/ethyl acetate v/v: 90/10); mp: $150-151{ }^{\circ} \mathrm{C}$.

${ }^{1} \mathrm{H}$ NMR $\left(\mathrm{CDCl}_{3}, 300 \mathrm{MHz}\right) \delta: 3.12-$ $3.28\left(\mathrm{~m}, 2 \mathrm{H}, \mathrm{H}_{3}\right), 3.79(\mathrm{~s}, 1 \mathrm{H}, \mathrm{NH}), 5.79-5.83$ $\left(\mathrm{m}, 1 \mathrm{H}, \mathrm{H}_{2}\right), 6.89-7.76(\mathrm{~m}, 13 \mathrm{H}, \mathrm{H}$ aromatic protons). ${ }^{13} \mathrm{C} \mathrm{NMR}\left(\mathrm{CDCl}_{3}, 75 \mathrm{MHz}\right) \delta: 36.7$ $\left(\mathrm{CH}_{3}\right) ; 55.4 \quad\left(\mathrm{C}_{2}\right) ; 69.3 \quad\left(\mathrm{C}_{3}\right) ; 114.3 \quad\left(\mathrm{C}_{18}\right.$, $\left.\mathrm{C}_{20}\right) ; 114.8\left(\mathrm{C}_{9}\right) ; 116.2\left(\mathrm{C}_{12}\right) ; 120.4\left(\mathrm{C}_{7}\right) ; 120.8$ $\left(\mathrm{C}_{10}\right) ; 124.2\left(\mathrm{C}_{14}\right) ; 127.1\left(\mathrm{C}_{6}\right) ; 132.7\left(\mathrm{C}_{17}, \mathrm{C}_{21}\right)$; $133.6\left(\mathrm{C}_{8}\right) ; 135.7\left(\mathrm{C}_{15}\right) ; 136.5\left(\mathrm{C}_{13}\right) ; 136.8$ $\left(\mathrm{C}_{16}\right) ; 137.1\left(\mathrm{C}_{5 \mathrm{a}}\right) ; 145.0\left(\mathrm{C}_{9 \mathrm{a}}\right) ; 150.5\left(\mathrm{C}_{19}\right)$; $162.0\left(\mathrm{C}_{11}\right) ; 171.0\left(\mathrm{C}_{4}\right)$. Mass $(\mathrm{m} / \mathrm{z})=335 . \mathrm{M}^{+}$ $=335$ (35.61); M+1 = $336(35.61) ; \mathrm{m} / \mathrm{Z}(\%)$; 219 (100); 218 (76); 91 (32.53); 77 (25.4); 52 (41.77).

(o-chlorophenyl)-4-phenyl-7-methyl-2,3dihydroxy-1,5-benzodiazepine $15 f$

From 1-phenyl-3-o-chlorophenylpropenone 14e (8.49 g, $35 \mathrm{mmol})$ and 4methyl-o-phenylenediamine $\mathbf{7 b}(5.13 \mathrm{~g}, 42$ mmol) was obtained 15f (7.95 g; 65\%) as yellow crystals; $\mathrm{R}_{\mathrm{f}}: 0.7$ (hexane/ethyl acetate v/v: 90/10); . mp: $122-123{ }^{\circ} \mathrm{C}$.

${ }^{1} \mathrm{H}$ NMR $\left(\mathrm{CDCl}_{3}, 300 \mathrm{MHz}\right) ; \delta: 2.23$ (s, 3H, $\mathrm{CH}_{3}$ ), 2.99-3.13 (m, 2H, $\left.\mathrm{H}_{3}\right), 3.64$ (s, $1 \mathrm{H}, \mathrm{NH}), 5.60-5.63\left(\mathrm{~m}, 1 \mathrm{H}, \mathrm{H}_{2}\right) ; 6.56-7.60$ (m, 12H, aromatic protons). ${ }^{13} \mathrm{C}$ NMR $\left(\mathrm{CDCl}_{3}, 75 \mathrm{MHz}\right)$ \&: $21.1\left(\mathrm{CH}_{3}\right) ; 34.9\left(\mathrm{C}_{3}\right)$; 
$67.1 \quad\left(\mathrm{C}_{2}\right) ; 120.8 \quad\left(\mathrm{C}_{9}\right) ; 124.6 \quad\left(\mathrm{C}_{6}\right) ; 128.1$ $\left(\mathrm{C}_{7}\right) ; 128.4\left(\mathrm{C}_{8}, \mathrm{C}_{21}\right) ; 132.2\left(\mathrm{C}_{19}\right) ; 132.7\left(\mathrm{C}_{18}\right)$; $134.7\left(\mathrm{C}_{20}\right) ; 136.6\left(\mathrm{C}_{12}, \mathrm{C}_{14}\right) ; 138.1\left(\mathrm{C}_{11}, \mathrm{C}_{15}\right)$; $138.6\left(\mathrm{C}_{13}\right) ; 138.8\left(\mathrm{C}_{17}\right) ; 139.2\left(\mathrm{C}_{10}\right) ; 140$ $\left(\mathrm{C}_{5 \mathrm{a}}\right) ; 141.2\left(\mathrm{C}_{16}\right) ; 142.1\left(\mathrm{C}_{9 \mathrm{a}}\right) ; 166,40\left(\mathrm{C}_{4}\right)$.

Mass $(\mathrm{m} / \mathrm{z})=346 . \mathrm{M}^{+}=346(46) ; \mathrm{M}+2=348$ (15); $\mathrm{m} / \mathrm{Z}(\%): 232$ (100); $231(65.81) ; 77$ (38.32).

(2-chloro-5-nitrophenyl)-4-phenyl-2,3dihydroxy-1,5-benzodiazepine $15 \mathrm{~g}$

From 1-phenyl-3-(2-chloro-5nitrophenyl)propenone (10.07 g, $35 \mathrm{mmol}$ ) and o-phenylene diamine 7a (4.54 g, 42 mmol) was obtained $\mathbf{1 5 g}(8.70 \mathrm{~g} ; 72 \%)$ as yellow crystal; $\mathrm{R}_{\mathrm{f}} 0.7$ (hexane/ethyl acetate v/v: 80/20); mp: $166-167{ }^{\circ} \mathrm{C}$.

${ }^{1} \mathrm{H}$ NMR $\left(\mathrm{CDCl}_{3}, 300 \mathrm{MHz}\right) \delta: 3.16$ (d, $\left.2 \mathrm{H}, \mathrm{H}_{3}, \mathrm{~J}=6 \mathrm{~Hz}\right), 3.71(\mathrm{~s}, 1 \mathrm{H}, \mathrm{NH}), 5.74-$ $5.79\left(\mathrm{~m}, 1 \mathrm{H}, \mathrm{H}_{2}\right), 6.85-7.88(\mathrm{~m}, 12 \mathrm{H}$, aromatic protons).

${ }^{13} \mathrm{C} \mathrm{NMR}\left(\mathrm{CDCl}_{3}, 75 \mathrm{MHz}\right) \delta: 33.3$ $\left(\mathrm{C}_{3}\right) ; 67.5\left(\mathrm{C}_{2}\right) ; 120.8\left(\mathrm{C}_{9}\right) ; 124.1\left(\mathrm{C}_{6}\right) ; 128.2$ $\left(\mathrm{C}_{7}\right) ; 128.7\left(\mathrm{C}_{19}\right) ; 129.1\left(\mathrm{C}_{21}\right) ; 132.7\left(\mathrm{C}_{8}\right)$; $133.2\left(\mathrm{C}_{12}, \mathrm{C}_{14}\right) ; 134.2\left(\mathrm{C}_{11}, \mathrm{C}_{15}\right) ; 138.2\left(\mathrm{C}_{13}\right)$; $139.2\left(\mathrm{C}_{10}\right) ; 139.8\left(\mathrm{C}_{5 \mathrm{a}}\right) ; 140.3\left(\mathrm{C}_{17}\right) ; 143.2$ $\left(\mathrm{C}_{16}\right) ; 143.7\left(\mathrm{C}_{9 \mathrm{a}}\right) ; 146.7\left(\mathrm{C}_{20}\right) ; 167.1\left(\mathrm{C}_{4}\right)$. Mass $(\mathrm{m} / \mathrm{z})=377 . \mathrm{M}^{+}=377(29.67) ; \mathrm{M}+2=$ 379 (10.24); M+1 = $378.1(10.10) ; \mathrm{m} / \mathrm{z}(\%)$ : 194 (100); 118,9 (33.21); 91 (16.41); 77 (14.88).

(2-chloro-5-nitrophenyl)-4-phenyl-7-methyl2,3-dihydroxy-1,5-benzodiazepine $15 \mathrm{~h}$ From 1-phenyl-3-(2-chloro-5nitrophenyl)propenone (10.07 g, $35 \mathrm{mmol})$ and 4-methyl-o-phenylenediamine $7 \mathbf{b}(5.13 \mathrm{~g}$, $42 \mathrm{mmol}$ ) was obtained 15h (10.55 g; 77\%) as yellow crystals; $\mathrm{R}_{\mathrm{f}}$ : 0.6 (hexane/ethyl acetate v/v: 80/20); mp: $200-201{ }^{\circ} \mathrm{C}$.

${ }^{1} \mathrm{H} \mathrm{NMR}\left(\mathrm{CDCl}_{3}, 300 \mathrm{MHz}, \delta: 2.23\right.$ (s, $\left.3 \mathrm{H}, \mathrm{CH}_{3}\right) ; 3.13\left(\mathrm{~d}, 2 \mathrm{H}, \mathrm{H}_{3}, \mathrm{~J}=6 \mathrm{~Hz}\right) ; 3.69(\mathrm{~s}$, $1 \mathrm{H}, \mathrm{NH})$; 5.70-5.67 (m, $\left.1 \mathrm{H}, \mathrm{H}_{2}\right) ; 6.85-7.88$ $\left(\mathrm{m}, 11 \mathrm{H}, \mathrm{H}\right.$ aromatic protons). ${ }^{13} \mathrm{C} \mathrm{NMR}$ $\left(\mathrm{CDCl}_{3}, 75 \mathrm{MHz}\right)$ \&: $20.1\left(\mathrm{CH}_{3}\right) ; 32.1\left(\mathrm{C}_{3}\right)$; $65.7\left(\mathrm{C}_{2}\right) ; 118.7\left(\mathrm{C}_{9}\right) ; 126.1\left(\mathrm{C}_{19}\right) ; 127.3\left(\mathrm{C}_{6}\right)$; $129.1\left(\mathrm{C}_{21}\right) ; 129.8\left(\mathrm{C}_{7}\right) ; 129.9\left(\mathrm{C}_{8}\right) ; 131.2$ $\left(\mathrm{C}_{12}, \mathrm{C}_{14}\right) ; 132.6\left(\mathrm{C}_{11}, \mathrm{C}_{15}\right) ; 135.2\left(\mathrm{C}_{13}\right) ; 138.2$ $\left(\mathrm{C}_{10}\right) ; 140.1\left(\mathrm{C}_{17}\right) ; 141.6\left(\mathrm{C}_{5 \mathrm{a}}\right) ; 143.6\left(\mathrm{C}_{9 \mathrm{a}}\right)$; $145.2\left(\mathrm{C}_{16}\right) ; 145.6\left(\mathrm{C}_{20}\right) ; 167.1\left(\mathrm{C}_{4}\right)$.

Mass $(\mathrm{m} / \mathrm{z})=391 . \mathrm{M}^{+}=391(29.74)$; $\mathrm{M}+2=393$ (9.15); M+1 = 392 (8.16); m/z (\%): 208 (100); 207 (48.95); 133 (24.59); 91 (6.22); 77 (20.69).
Benzimidazoles 16 - General method

A solution of 2,3-dihydro-1,5benzodiazepine $15(1 \mathrm{~g})$ and potassium carbonate $(3 \mathrm{~g})$ in $10 \mathrm{~mL}$ of dry dimethylformamide (DMF) was refluxed for $24 \mathrm{~h}$. After cooling to room temperature, 20 $\mathrm{mL}$ of water was added. The organic layer was extracted with ethyl acetate $(2 \times 50 \mathrm{~mL})$, dried with magnesium sulfate, filtered and concentrated under reduced pressure. The residue was purified by column chromatography on silica gel.

phenyl-1H-benzimidazole 16 a

From 2-(2-chloro-5-nitrophenyl)-4phenyl-2,3-dihydroxy-1,5-benzodiazepine $\mathbf{1 5 g}$ (1 g, $2.6 \mathrm{mmol})$ and potassium carbonate (1.8 $\mathrm{g}, 13 \mathrm{mmol})$ was obtained 16a $(0.25 \mathrm{~g} ; 50 \%)$; $\mathrm{R}_{\mathrm{f}}: 0.5$ (hexane/ethyl acetate $\mathrm{v} / \mathrm{v}: 50 / 50$ ); mp: 250-251 ${ }^{\circ} \mathrm{C}$.

Or from 2,4-diphenyl-2,3-dihydro-1,5benzodiazepine 15a (1 g, $3.3 \mathrm{mmol})$ and potassium carbonate $(2.28 \mathrm{~g}, 16.5 \mathrm{mmol})$ was obtained 16a $(0.29 \mathrm{~g} ; 45 \%) ; \quad \mathrm{R}_{\mathrm{f}}: 0.5$ (hexane/ethyl acetate v/v: 50/50); mp: 250$251{ }^{\circ} \mathrm{C}$ Or from 4-Phenyl-2-Thienyl-2,3dihydro-1,5-benzodiazepine 15d (1 g, 3.29 mmol) and potassium carbonate $(2.27 \mathrm{~g}, 16.45$ mmol) was obtained 16a $(0.38 \mathrm{~g} ; 60 \%) ; \mathrm{R}_{\mathrm{f}}$ : 0.5 (hexane/ethyl acetate v/v: 50/50); mp: 250-251 ${ }^{\circ} \mathrm{C}$.

${ }^{1} \mathrm{H} \mathrm{NMR}\left(\mathrm{CDCl}_{3}, 300 \mathrm{MHz}\right) ; \delta: 7.10-$ $7.92(\mathrm{~m}, 15 \mathrm{H}$, aromatic protons); $12.80(\mathrm{~s}, 1 \mathrm{H}$, $\mathrm{NH}) .{ }^{13} \mathrm{C} \mathrm{NMR}\left(\mathrm{CDCl}_{3}, 75 \mathrm{MHz}\right) \delta: 111.4$ $\left(\mathrm{C}_{4}\right) ; 113.1 \quad\left(\mathrm{C}_{7}\right) ; 124.3 \quad\left(\mathrm{C}_{6}\right) ; 127.7\left(\mathrm{C}_{13}\right)$; $128.1\left(\mathrm{C}_{9}\right) ; 128.8\left(\mathrm{C}_{11}\right) ; 130.1\left(\mathrm{C}_{5}\right) ; 130.9\left(\mathrm{C}_{10}\right.$, $\left.\mathrm{C}_{12}\right) ; 131.7\left(\mathrm{C}_{8}\right) ; 138\left(\mathrm{C}_{3 \mathrm{a}}\right) ; 143.8\left(\mathrm{C}_{7 \mathrm{a}}\right) ; 151.3$ $\left(\mathrm{C}_{2}\right)$. Mass $(\mathrm{m} / \mathrm{z})=194 \cdot \mathrm{M}^{+}=194(100) ; \mathrm{M}+1$ $=195(15.16) ; \mathrm{m} / \mathrm{z}(\%): 193(27.21) ; 102.9$ (11.39); 91 (16.41); 77 (16.75); 51 (10.85).

phenyl-5-méthyl-1H-benzimidazole $16 b$

From 7-methyl-2,4-diphenylbenzodiazepine (1 g, $3.2 \mathrm{mmol}$ ) and potassium carbonate $(2.21 \mathrm{~g}, 16 \mathrm{mmol})$ was obtained $16 \mathrm{~b}$ $(0.27 \mathrm{~g} ; 40 \%)$; Rf: 0.2 (hexane/ethyl acetate 95/5); mp : 220-221 ${ }^{\circ} \mathrm{C}$.

Or from 7-methyl-4-phenyl-2-(2chlorophenyl) benzodiazepine (1 g, 2.88 mmol) and potassium carbonate (1.99 g, 14.40 mmol) was obtained $16 \mathrm{~b}(0.33 \mathrm{~g} ; 55 \%)$; Rf: 0.2 (hexane/ethyl acetate 95/5); mp : 220-221 ${ }^{\circ} \mathrm{C}$. 
${ }^{1} \mathrm{H} \mathrm{NMR}\left(\mathrm{CDCl}_{3}, 300 \mathrm{MHz}\right) \delta: 2.42(\mathrm{~s}$, $\mathrm{CH}_{3}$ ); 7.01-8.17 (m, 8H, aromatic protons); $12.80(\mathrm{~s}, 1 \mathrm{H}, \mathrm{NH}) .{ }^{13} \mathrm{C}$ NMR $\left(\mathrm{CDCl}_{3}, 75\right.$ $\mathrm{MHz}) \delta$ : $21.4\left(\mathrm{CH}_{3}\right) ; 123.6\left(\mathrm{C}_{7}\right) ; 124.5\left(\mathrm{C}_{4}\right)$; $124.5\left(\mathrm{C}_{6}\right) ; 126.9\left(\mathrm{C}_{13}\right) ; 127.6\left(\mathrm{C}_{9}\right) ; 128.8$ $\left(\mathrm{C}_{11}\right) ; 128.9\left(\mathrm{C}_{5}\right) ; 130.2\left(\mathrm{C}_{10}, \mathrm{C}_{12}\right) ; 130.6\left(\mathrm{C}_{8}\right)$; $\left.130.9 \mathrm{C}_{3 \mathrm{a}}\right) ; 150.9\left(\mathrm{C}_{7 \mathrm{a}}\right) ; 162.3\left(\mathrm{C}_{2}\right)$. Mass $(\mathrm{m} / \mathrm{z}): 208 \mathrm{M}^{+}=208(29.68) ; \mathrm{M}+1=209$ (73.85); $\mathrm{M}+2=210(12.09) ; \mathrm{m} / \mathrm{z}(\%): 191$ (45.78); 189 (100); 145 (45.22); 131 (11); 40 (30.95).

\section{2-(paratolyl)-1H-benzimidazole $16 \mathrm{c}$}

From 2-thienyl-4-(p-tolyl) benzodiazepine ( $1 \mathrm{~g}, \quad 3.14 \mathrm{mmol})$ and potassium carbonate $(2.17 \mathrm{~g}, 15.70 \mathrm{mmol})$ was obtained $16 \mathrm{c}(0.23 \mathrm{~g} ; 35 \%)$; Rf: 0.5 (hexane/ethyl acetate 25/75);mp: $180-181{ }^{\circ} \mathrm{C}$.

${ }^{1} \mathrm{H} \mathrm{NMR}\left(\mathrm{CDCl}_{3}, 300 \mathrm{MHz}\right) \delta: 2.45$ (s, $\left.3 \mathrm{H}, \mathrm{CH}_{3}\right) ; \quad 6.80-8.15(\mathrm{~m}, \quad 8 \mathrm{H}$, aromatic protons); 12.80 (s, $1 \mathrm{H}, \quad \mathrm{NH}) .{ }^{13} \mathrm{C} \quad \mathrm{NMR}$ $\left(\mathrm{CDCl}_{3}, 75 \mathrm{MHz}\right) \delta: 20.3\left(\mathrm{CH}_{3}\right) ; 112.7\left(\mathrm{C}_{4}\right.$, $\left.\mathrm{C}_{7}\right) ; 118.6\left(\mathrm{C}_{5}, \mathrm{C}_{6}\right) ; 124.2\left(\mathrm{C}_{9}, \mathrm{C}_{13}\right) ; 128.5$ $\left(\mathrm{C}_{8}\right) ; 130.5\left(\mathrm{C}_{10}, \mathrm{C}_{12}\right) ; 136.5\left(\mathrm{C}_{11}\right) ; 143.6\left(\mathrm{C}_{3 \mathrm{a}}\right.$, $\left.\mathrm{C}_{7 \mathrm{a}}\right) ; 154.2\left(\mathrm{C}_{2}\right)$. Mass $(\mathrm{m} / \mathrm{z}): 208 \mathrm{M}^{+}=208$ (30.25); $\mathrm{M}+1=209$ (67.63); $\mathrm{M}+2=210$ (13.20); m/z (\%): 191 (40.48); 189 (100); 145 (45.72); 131 (15.12); 40 (26.35).

orthohydroxyphenyl-1H-benzimidazole $16 d$

From 4-(o-hydroxyphenyl-2-(pmethoxyphenyl) benzodiazepine (1 g, 2.9 $\mathrm{mmol})$ and potassium carbonate $(1.8 \mathrm{~g}, 13$ mmol) was obtained $16 \mathrm{c}(0.21 \mathrm{~g} ; 35 \%)$; Rf: 0.5 (hexane/ethyl acetate 80/20); mp: 198-199 ${ }^{\circ} \mathrm{C}$.

${ }^{1} \mathrm{H}$ NMR $\left(\mathrm{CDCl}_{3}, 300 \mathrm{MHz}\right) \delta: 6.79$ $7.70(\mathrm{~m}, 8 \mathrm{H}$, aromatic protons); $9.80(\mathrm{~s}, 1 \mathrm{H}$, $\mathrm{OH}) ; 12.80$ (s, $1 \mathrm{H}, \mathrm{NH}) .{ }^{13} \mathrm{C} \mathrm{NMR}\left(\mathrm{CDCl}_{3}, 75\right.$ $\mathrm{MHz}) \delta: 115.2\left(\mathrm{C}_{4}, \mathrm{C}_{7}\right) ; 118.5\left(\mathrm{C}_{10}\right) ; 120.1$ $\left(\mathrm{C}_{8}\right) ; 122.6\left(\mathrm{C}_{12}\right) ; 124\left(\mathrm{C}_{5}, \mathrm{C}_{6}\right) ; 128\left(\mathrm{C}_{13}\right)$; $132.8\left(\mathrm{C}_{11}\right) ; 138.4\left(\mathrm{C}_{3 \mathrm{a}}, \mathrm{C}_{7 \mathrm{a}}\right) ; 152.9\left(\mathrm{C}_{2}\right) ; 155.2$ $\left(\mathrm{C}_{9}\right)$. Mass (m/z): $210 \mathrm{M}^{+}=210(35.21) ; \mathrm{M}+1$ $=211(70.15) ; \mathrm{M}+2=212(10.40) ; \mathrm{m} / \mathrm{z}(\%)$ : 191 (55.66); 189 (100); 145 (40.25); 131 (14); 40 (28.24).

\section{RESULTS AND DISCUSSION}

For several years, our research team is interested in the study of the heteroatomic chains of $N^{\prime}$-thioacylamidines (Figure 2) and the synthesis of benzimidazole derivatives. The synthesis of $N^{\prime}$-thioacylamidine intermediaries are widely established. These compounds are easily accessible (Téa et al.,
1986) and are subjected of several physicochimique studies (Chehna et al., 1989).

The obtained results are highlighted as follow: dienic (Téa et al., 1983, 1985, 1986a, 1986b; Chehna et al., 1987), nucleophilic (Meslin et al., 1974, 1975) electrophilic (Meslin et al., 1974, 1975; Guemas et al., 1982) and dipolaroplic characters (De Boer et al., 1967).

Theoretical calculations were made with the imidinium salts in order to determine their reaction ability and stability. The results of the theoretical calculations MNDO, AM1 and PM3 conducted on various imidinium salts confirmed their electrophilic character. The values of the orbital coefficients $2 \mathrm{Pz}$ and the calculated loads using the method AM1 were indicated in the Figure 3.

These results showed that carbons $\mathrm{C} 1$ and C3 would be subjected to nucleophilic attacks.

In our search for new benzimidazole derivatives, two main synthetic approaches were used for the preparation of benzimidazole derivatives. In first previous works, we used amidinium salts as starting material. Recently, we developed in our laboratory, a study of the reactivity of amidinium salts against binucleophiles like $o$ phenylenediamine. Thus, $o$-phenylenediamine 7 and amidinium salt 8 were condensed at 5 ${ }^{\circ} \mathrm{C}$ in ethanol. The reaction afforded 2-phenyl1,3-benzimidazole 9 and 2-phenyl-1,3,5benzotriazepine 10 (Scheme 1).

The 2-phenyl-1,3-benzimidazole 9 resulted from a double attack of $o$ phenylenediamine to the $\mathrm{C} 3$ of amidinium salt and the 2-phenyl-1,3,5-benzotriazepine $\mathbf{1 0}$ from a double attack of $o$-phenylenediamine to the $\mathrm{C} 3$ and $\mathrm{C} 1$ of amidinium salt. At this temperature, the 2-phenyl-1,3-benzimidazole 9 was formed as the major product which was isolated in moderate yield $(60 \%)$.

Attempt to conduct the same reaction between $o$-phenylenediamine and 3-thioalkyl or 3-thioarylamidinium salt was unsuccessful. Surprisingly, Sissouma et al. (2004) obtained the 1,3-benzimidazole $\mathbf{1 1}$ and the 2thiosubstituted benzimidazole 12 (Scheme 2).

The yield of compounds $\mathbf{1 1}$ and $\mathbf{1 2}$ depended on the temperature. By raising the temperature, the yield of compound $\mathbf{1 1}$ increased while that of compound $\mathbf{1 2}$ 


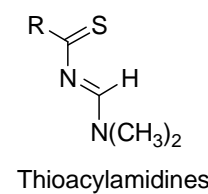

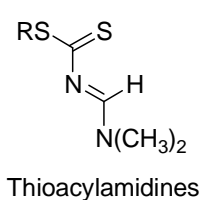

Thioacylamidines

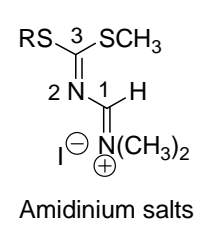

Figure 2: The heteroatomic chains of $N^{\prime}$-thioacylamidines.

decreased. At room temperature, the yield of 11 became important. We expected to obtain the benzotriazepine ring but we did not in spite of our numerous attempts. The formation of 1,3-benzimidazole could be explained by the formation of a seven member ring, 2thiosubstituted-1,3,5-benzotriazepine intermediate resulting by double attack on $\mathrm{C} 1$ and C3 (Scheme 3).

The 2-thiosubstituted-1,3,5benzotriazepine must evolve by intermolecular nucleophilic attack. This mechanism was similar to that proposed by Ugi et al. (1986) in the synthesis of $\beta$-lactams (Scheme 3).

The second original method was based on the use of benzodiazepine as intermediate compounds. Although many methods for synthesizing benzodiazepine ring systems they continued to receive a great deal attention (Kidwai et al., 2004; Kusanur et al., 2004; Kumar and Joshi, 2007; Sanghetti et al., 2007).

Generally, benzodiazepines are synthesized by the condensation of $o$ phenylenediamine with $\alpha, \beta$-unsaturated carbonyl compounds, haloketones or ketones. In the synthesis process, we used chalcone as starting material. Thus, chalcones 14 were prepared by using Claisen-Schmidt condensation with appropriate substituted benzaldehydes and substituted aromatic ketone (acetophenone) in $\mathrm{KOH} / \mathrm{EtOH}$ solution (Scheme 4). Physical characteristics and yields of compounds 14 were summarized in Table 1. Indeed, the reaction of binucleophiles like $o$-phenylenediamine with $\alpha, \beta$-unsaturated carbonyl compounds $\mathbf{1 4}$ under reflux in the presence of triethylamine in ethanol or methanol afforded compounds $\mathbf{1 5}$ in moderate to good yields (36-77\%). The reaction was carried out in dark in order to exclude any influence of light with a possible oxidation of $o$-phenylenediamine (Braulio et al., 1997). Benzodiazepine was isolated as only reaction product (Scheme 4).

The yields of the reactions were improved by using methanol as solvent instead of ethanol.

The main features of ${ }^{1} \mathrm{H}$ NMR spectra in $\mathrm{CDCl}_{3}$ of the isolated compounds $\mathbf{1 5}$ are reported in Table 2 . The ${ }^{1} \mathrm{H}$ NMR analysis of compounds 15b, 15c and 15d showed that H3 protons were not equivalent. In compound $\mathbf{1 5 b}$, these protons were appeared as doublet dedoublet (dd) at 3.04 and $3.32 \mathrm{ppm}$; they were coupling with $\mathrm{H} 2$ proton $(5.15 \mathrm{ppm}, \mathrm{dd})$. The constants coupling were: ${ }^{2} \mathbf{J}_{\mathrm{H} 3 \alpha-\mathrm{H} 3 \beta}=15$ $\mathrm{Hz},{ }^{3} \mathrm{~J}_{\mathrm{H} 3 \alpha-\mathrm{H} 2}=9 \mathrm{~Hz}$ and ${ }^{3} \mathrm{~J}_{\mathrm{H} 3 \mathrm{\beta}-\mathrm{H} 2}=6 \mathrm{~Hz}$. This spectrum is characteristic of AMX system which is shown in Figure 4. The yields of benzodiazepines, $1 \mathrm{H}$ chemical shifts (ppm) and $1 \mathrm{H}-1 \mathrm{H}$ coupling constants $(\mathrm{J}$ in $\mathrm{Hz}$ ) of compounds 15 in $\mathrm{CDCl}_{3}$ were summarized in Table 2.

The analysis of the AMX system corresponding to the protons $\mathrm{H} 2$ and $\mathrm{H} 3$ gave the couplings represented in Figure 4. To confirm the structure of compounds $\mathbf{1 5}$, we realized the X-ray structure analysis (Bibila et al., 2010) of compound $\mathbf{1 5 b}$ which is shown on Figure 5 and the crystal data is summarized 
in Table 3. The molecular of benzodiazepine is crystallized in monoclinic system. The ring system of benzodiazepine adopted a distorted boat conformation. The benzene ring of this system formed dihedral angles of 89.69 (12) and 48.82 (12) with those of the phenol and methoxyphenyl substituent respectively. The dihedral angle between the benzene rings was 49.61 (11). An intramolecular $\mathrm{O}-\mathrm{H}-----\mathrm{N}$ hydrogen bond generated an $\mathrm{S}(6)$ ring. Treatment of compounds $\mathbf{1 5}$ with potassium carbonate in dimethylformamide (DMF) under reflux, afforded 2-substituted benzimidazole 16 in moderate yields (4060\%) and quinoxaline 17 (Scheme 4).
The spectroscopic analysis of protons showed the disappearance of protons $\mathrm{H} 2$ and H3 observed in compounds $\mathbf{1 5}$. We also noted an echo zone concentrated in the aromatic area with a decrease of protons. The benzimidazolic structures that we proposed were confirmed by mass spectrometry data. To explain the formation of the benzimidazole we proposed the following mechanism: 2,3dihydro-benzodiazepine 15 underwent $\mathrm{K}_{2} \mathrm{CO}_{3}$ attacked to give an amidure ion which, by intramolecular reaction, reacted on the carbon $\mathrm{C} 4$ of the imine function. The loss of styrene afforded the benzimidazole 16 (Scheme 5).

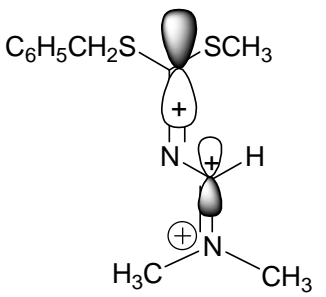

$\mathbf{C}_{1}=0.491, \mathrm{q}_{1}=0.387$

$\mathbf{C}_{3}=-0.593, \mathrm{q}_{3}=-0.015$

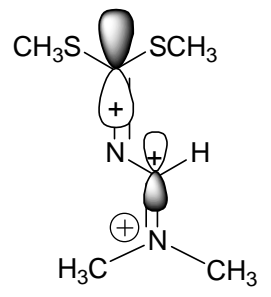

$\mathbf{C}_{1}=0.389, \mathrm{q}_{1}=0.483$

$\mathbf{C}_{3}=-0.588, q_{3}=-0.012$

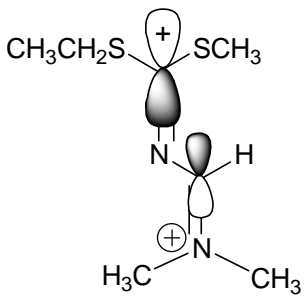

$\mathbf{C}_{1}=-0.485, \mathrm{q}_{1}=0.388$

$\mathbf{C}_{3}=0.590, q_{3}=-0.012$

$\mathrm{q}_{i}$ is the charge and $\mathrm{C}_{\mathrm{i}}$ the orbital coefficients in the vacant orbital

Figure 3: Values of the orbital coefficients $2 \mathrm{Pz}$.

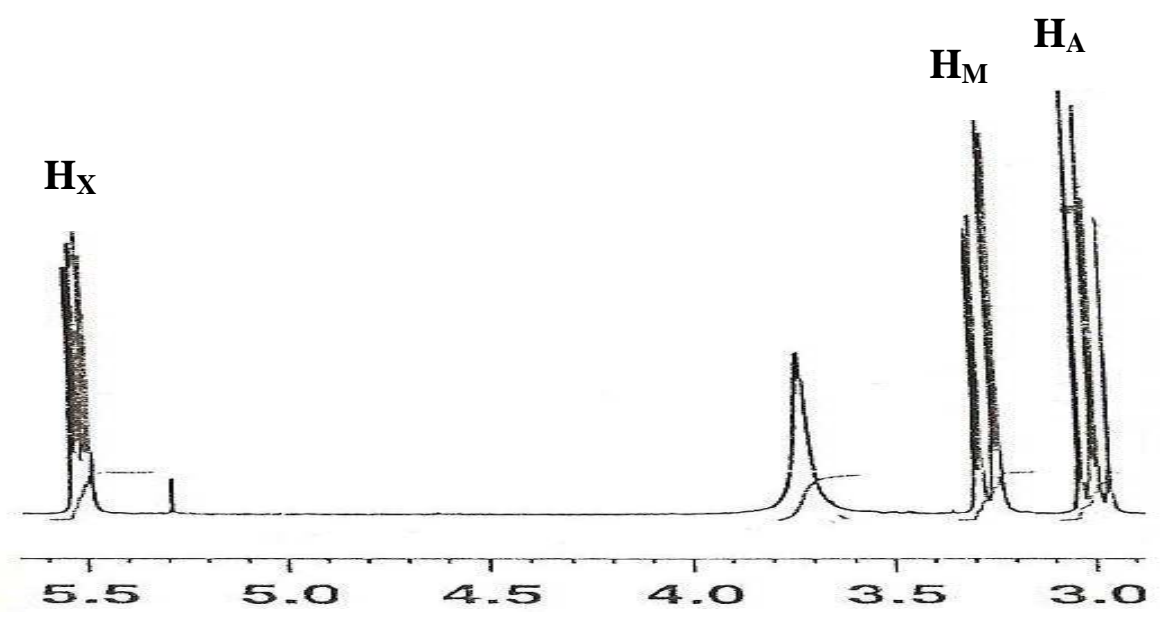

Figure 4: AMX spectrum of compound 15c. 


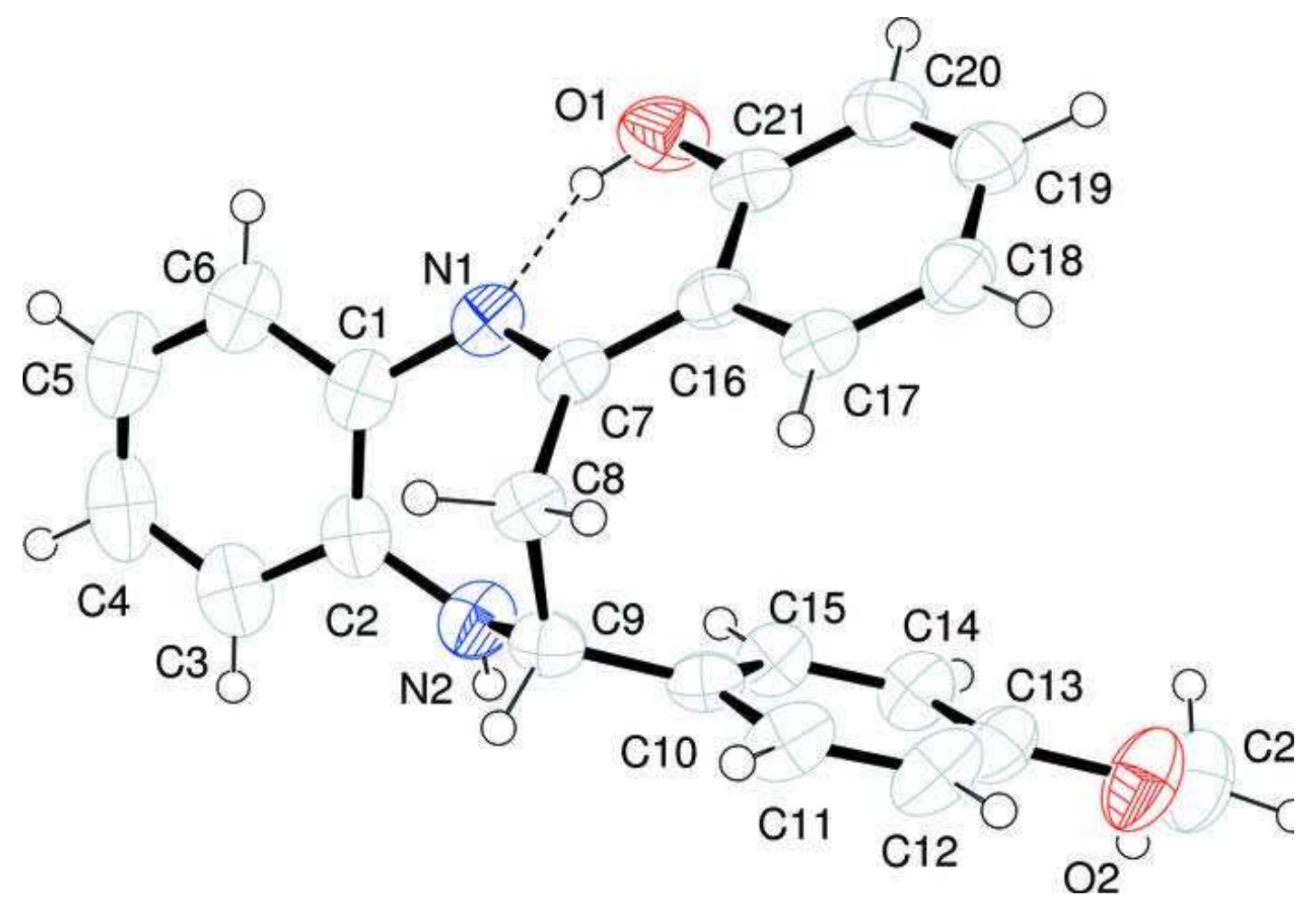

Figure 5: X-ray crystal structure of compound $15 \mathrm{~b}$.

Table 1: Physical characteristics and yields of compounds 14.

\begin{tabular}{lllll}
\hline Compounds & $\mathbf{R}^{\mathbf{2}}$ & $\mathbf{R}^{\mathbf{3}}$ & Yields $(\boldsymbol{\%})$ & $\mathbf{m p .}\left({ }^{\circ} \mathbf{C}\right)$ \\
\hline 14a & Phenyl & Phenyl & 71 & $56-57$ \\
14b & $p$-Methoxyphenyl & $o$-Hydroxyphenyl & 85 & $118-119$ \\
$\mathbf{1 4 c}$ & Thienyl & $p$-Tolyl & 72 & $81-82$ \\
$\mathbf{1 4 d}$ & Thienyl & Phenyl & 78 & $121-122$ \\
$\mathbf{1 4 e}$ & $o$-chlorophenyl & Phenyl & 70 & $111-112$ \\
$\mathbf{1 4 f}$ & 2-Chloro-5-nitrophenyl & Phenyl & 68 & $96-97$ \\
\hline
\end{tabular}<smiles>Nc1ccccc1N</smiles>

7<smiles>CCOS(=O)(=O)c1ccccc1</smiles>

8

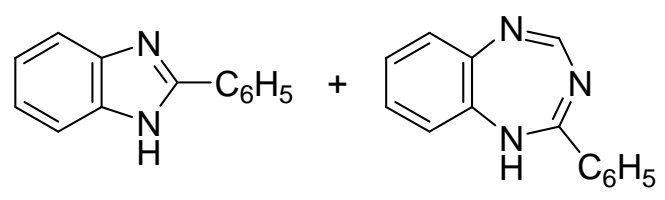

9
10

Scheme 1 
Table 2: Physical characteristics, ${ }^{1} \mathrm{H}$ chemical shifts (ppm) and ${ }^{1} \mathrm{H}-{ }^{1} \mathrm{H}$ coupling constants ( $\mathrm{J}$ in $\mathrm{Hz}$ ) of compounds 15 in $\mathrm{CDCl}_{3}$.

\begin{tabular}{|c|c|c|c|c|c|c|c|}
\hline Compounds & $\mathbf{R}$ & $\mathbf{R}^{2}$ & $\mathbf{R}^{3}$ & Yields (\%) & $\mathbf{P F}^{\circ} \mathbf{C}$ & $\delta(\mathbf{p p m})$ & $\mathbf{J}(\mathbf{H z})$ \\
\hline 15a & $\mathrm{H}$ & Phenyl & Phenyl & 41 & & $\begin{array}{l}3.06-3.08(\mathrm{~m}, \mathrm{H} 3) \\
5.87-5.88(\mathrm{~m}, \mathrm{H} 2)\end{array}$ & \\
\hline $15 b$ & $\mathrm{H}$ & $p$-Metoxyphenyl & $o$-Hydroxyphenyl & 36 & $141-142$ & $\begin{array}{l}3.04(\mathrm{dd}, \mathrm{H} 3 \alpha) \\
3.32(\mathrm{dd}, \mathrm{H} 3 \beta) \\
5,15(\mathrm{dd}, \mathrm{H} 2)\end{array}$ & $\begin{array}{l}{ }^{3} \mathrm{~J}_{\mathrm{H} 3 \alpha-\mathrm{H} 2}=9,{ }^{2} \mathrm{~J}_{\mathrm{H} 3 \alpha-\mathrm{H} 3 \beta}=15 \\
{ }^{3} \mathrm{~J}_{\mathrm{H} 3 \beta-\mathrm{H} 2}=6\end{array}$ \\
\hline $15 \mathrm{c}$ & $\mathrm{H}$ & Thienyl & $p$-Tolyl & 45 & 125 & $\begin{array}{l}2.99(\mathrm{dd}, \mathrm{H} 3 \alpha) \\
3.26(\mathrm{dd}, \mathrm{H} 3 \beta) \\
5,51(\mathrm{dd}, \mathrm{H} 2)\end{array}$ & $\begin{aligned}{ }^{3} \mathrm{~J}_{\mathrm{H} 3 \alpha-\mathrm{H} 2} & =9,{ }^{2} \mathrm{~J}_{\mathrm{H} 3 \alpha-\mathrm{H} 3 \beta}=13.2 \\
{ }^{3} \mathrm{~J}_{\mathrm{H} 3 \beta-\mathrm{H} 2} & =4.2\end{aligned}$ \\
\hline 15d & $\mathrm{H}$ & Thienyl & Phenyl & 52 & $133-134$ & $\begin{array}{l}3.06(\mathrm{dd}, \mathrm{H} 3 \alpha) \\
3.32(\mathrm{dd}, \mathrm{H} 3 \beta) \\
5.55(\mathrm{dd}, \mathrm{H} 2)\end{array}$ & $\begin{array}{l}{ }^{3} \mathrm{~J}_{\mathrm{H} 3 \alpha-\mathrm{H} 2}=9,{ }^{2} \mathrm{~J}_{\mathrm{H} 3 \alpha-\mathrm{H} 3 \beta}=12 \\
{ }^{3} \mathrm{~J}_{\mathrm{H} 3 \beta-\mathrm{H} 2}=6\end{array}$ \\
\hline $15 \mathrm{e}$ & $\mathrm{H}$ & $o$-Chlorophenyl & Phenyl & 75 & $150-151$ & $\begin{array}{l}3.12-3.28(\mathrm{~m}, \mathrm{H} 3) \\
5.79-5.83(\mathrm{~m}, \mathrm{H} 2)\end{array}$ & \\
\hline $15 f$ & $\mathrm{CH}_{3}$ & $o$-Chlorophenyl & Phenyl & 65 & $122-123$ & $\begin{array}{l}\text { 2.99-3.13 (m, H3), } \\
\text { 5.60-5.63 (m, H2) }\end{array}$ & \\
\hline $15 \mathrm{~g}$ & $\mathrm{H}$ & 2-Chloro-5-nitrophenyl & Phenyl & 71 & $167-168$ & $\begin{array}{l}3.16(\mathrm{~d}, \mathrm{H} 3) \\
5.74-5.79(\mathrm{~m}, \mathrm{H} 2)\end{array}$ & $\mathrm{J}=6$ \\
\hline $15 \mathrm{~h}$ & $\mathrm{CH}_{3}$ & 2-Chloro-5-nitrophenyl & Phenyl & 77 & $223-224$ & $\begin{array}{l}3.13(\mathrm{~d}, \mathrm{H} 3) \\
5.70-5.67(\mathrm{~m}, \mathrm{H} 2)\end{array}$ & $\mathrm{J}=6$ \\
\hline
\end{tabular}


Table 3: Crystal data of compound $15 \mathrm{~b}$.

\begin{tabular}{ll}
\hline $\mathrm{C}_{22} \mathrm{H}_{20} \mathrm{~N}_{2} \mathrm{O}_{2}$ & $\mathrm{~V}=3506.02(15) \AA^{3}$ \\
$\mathrm{Mr}=344.41$ & $\mathrm{Z}=8$ \\
Monoclinic, $\mathrm{C} 2=\mathrm{c}$ & $\mathrm{MoK} \alpha$ radiation \\
$\mathrm{a}=27.5064(5) \AA$ & $\mu=0.08 \mathrm{~mm}^{-1}$ \\
$\mathrm{~b}=7.3811(2) \AA$ & $\mathrm{T}=223 \mathrm{~K}$ \\
$\mathrm{c}=19.5038(4) \AA$ & $0.30 \times 0.20 \times 0.15 \mathrm{~mm}$ \\
$\beta=117.699(2)$ & 2836 reflections with $\mathrm{I}>3 \sigma(\mathrm{I})$ \\
\hline Data collection & Rint $=0.06$ \\
Nonius Kappa CCD diffractometer & \\
19187 measured reflections & \\
2507 independent reflections & 235 parameters \\
\hline Refinement & $\mathrm{H}$-atom parameters constrained \\
$\mathrm{R}[\mathrm{F} 2>2 \sigma(\mathrm{F} 2)]=0.055$ & $\Delta \rho$ max $=0.25$ e $\AA^{-3}$ \\
wR(F2) $=0.065$ & $\Delta \rho$ min $=-0.25$ e $\AA^{-3}$ \\
$\mathrm{~S}=1.04$ & \\
2507 reflections & \\
\hline
\end{tabular}

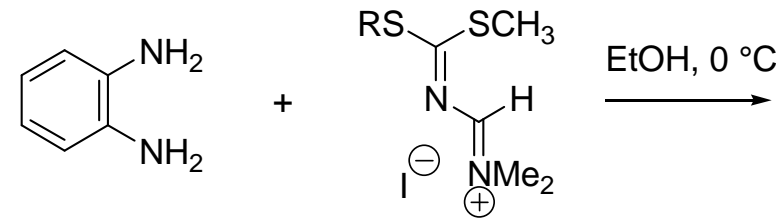

$\mathrm{R}=\mathrm{CH}_{3}, \mathrm{CH}_{3} \mathrm{CH}_{2}$ ou $\mathrm{C}_{6} \mathrm{H}_{5} \mathrm{CH}_{2}$<smiles>c1ccc2[nH]cnc2c1</smiles>

11<smiles>Bc1nc2ccccc2[nH]1</smiles>

12

\section{Scheme 2}

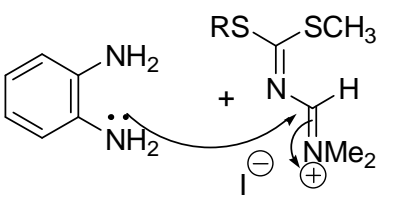

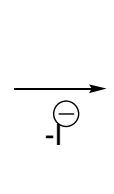<smiles></smiles><smiles>CO</smiles><smiles></smiles>

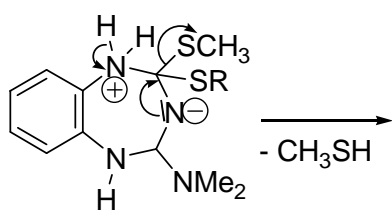

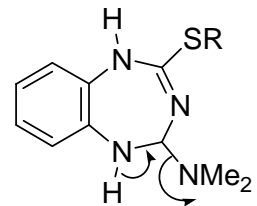<smiles>CNCCN(C)C</smiles><smiles>SC1=NCCN2CCNC1=N2</smiles><smiles>SC1=NC2Nc3ccccc3N12</smiles><smiles></smiles>

Scheme 3 


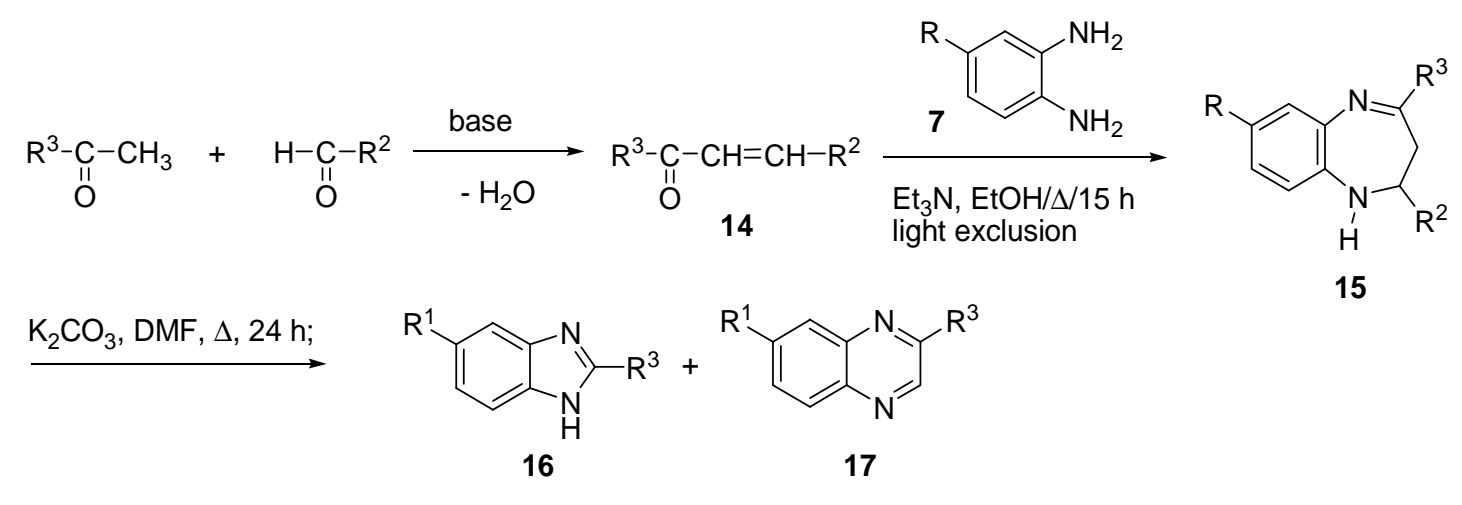

Scheme 4<smiles>[R]C1=C(C)Nc2ccc([13F])cc2NC1[R7]</smiles>

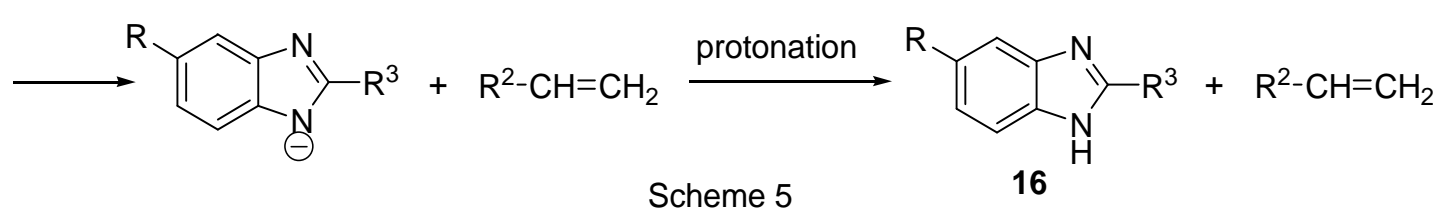

\section{Conclusion}

Through this work, we have shown the synthesis of substituted benzimidazoles by an original method via regression (Ring Contraction Rearrangement) of $1 \mathrm{H}-1,5-$ benzodiazepine under basic conditions. Structure elucidation of benzodiazepine and benzimidazole was made possible by NMR and mass spectrometry methods. We have also confirmed the benzodiazepine structure by realizing X-ray crystal structure analysis.

\section{ACKNOWLEDGMENTS}

We thank the Laboratory of Chemistry and Interdisciplinarity, synthesis, analysis, modeling (Chimie Et Interdisciplinarité, Synthèse, Analyse, Modélisation) (CEISAM), University of Nantes (France) for

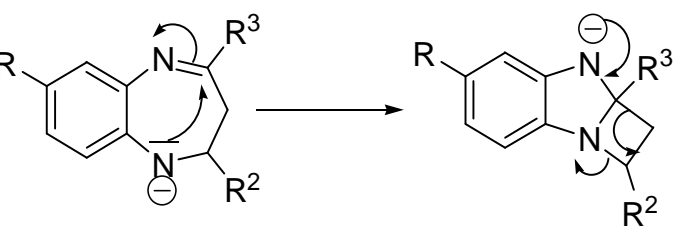

Scheme 5 spectroscopic analyses (NMR and Mass). Our thanks go towards the Laboratory of Crystallography and Molecular Physical appearance, UFR SSMT University Félix Houphouët Boigny (République de Côte d'Ivoire) for the X-ray spectral data.

\section{REFERENCES}

Al-Muhaimeed HS. 1997. A parallel-group comparison of Astemizole and Loratadine for the treatment of Perennial Allergic Rhinitis. J. Int. Med. Res., 25: 175-181.

Ashnagar A, Naseri N and Amini M. 2009. Synthesis of 5,5-diphenyl-2,4imidazolidinedione (Phenytoin), International Journal of ChemTech Research, 1(1): 47-52. 
Bibila MBY, Adjou A, Yapi MY, Bany GE, Kakou-Yao RCA. 2010. 2-[2-(4Methoxyphenyl)-2,3-dihydro-1H-1,5benzodiazepin-4-yl]phenol. Acta Cryst., E66: 087-088.

Braulio I, Ricaurte R, Jairo Q, Roberto M. 1997. Synthesis of substituted 1-benzyl2,4,6-triphenyl-2,3-dihydro-pyrazolo [3,4b] [1,4]diazepines Journal of Heterocyclic Chemistry, 34: 1131-1134.

Chehna M, Pradère JP, Guigant A. 1987. Selective synthesis of substituted $6 \mathrm{H}-1,3-$ Thiazines. Synthetic Commun., 17: 19711976.

Chehna M, Pradère JP, Téa CG, Quiniou H, Le Botlan D. 1989. Substituted 1-Thia-3aza-1,3-butadiene : Diels-Alder reaction with methylacrylate. Determination of activation parameters. Phosphorus Sulfur And Silicon, 42: 15-19.

De Boer TJ, Backer HJ. 1967. Organic Synthesis (Coll. vol. IV). John Wiley and Sons: New York; 250.

DeLong DC. 1984. Effect of enviroxime on rhinovirus infectionsin humans. In Microbiology, Leive L, Schlessinger D (eds). American Society for Microbiology: Washington, DC; 431-434.

Gardiner JM, Loyns CR, Burke A, Khan A, Mahmood N. 1995. Synthesis and HIV-1 inhibition of novel benzimidazole derivatives. Bioorg. Med. Chem. Lett., 5: 1251-1254.

Garuti L, Roberti M, Rossi T, Cermelli C, Portolani M, Malagoli M, Castelli M. 1998. Synthesis, antiviral and antiproliferative activity of some $\mathrm{N}$ benzenesulphonyl-2-(2-or 3-pyridylethyl)benzimidazoles, Anti-Cancer Drug Design, 13: 397-406.

Guemas JP, Lees M, Reliquet A, Villieras J. 1982. Addition of organometallics on $\alpha, \beta$ unsaturated thiocarbonyl coumpounds. Michael addition of lithium enolates on thioamide and dithiocarbamate vinylogs and thioarylformadine. Phosphorus and Sulfur, 12: 325-339.

Hazelton JC, Iddon B, Suschitzky H, Woolley LH. $\quad 1995 . \quad 2 H$-Benzimidazoles (Isobenzimidazoles). Part 10.1,2 Synthesis of Polysubstituted $o$-Phenylenediamines and their Conversion into Heterocycles, Particularly 2-Substituted Benzimidazoles with Known or Potential Anthelminthic Activity. Tetrahedron, 51: 10771-10794.

Kidwai M, Ruby MK and Venkataramanan R. 2004. A facile synthesis of substituted benzodiazepines using solid support. Chem. Heterocyclic Comp., 40: 631-634.

Kumar R, Joshi YC. 2007. Synthesis spectral studies and biological activity of $3 H-1,5-$ benzodiazepine derivatives. Arkivoc, XIII: 142-149.

Kusanur RA, Ghate M, Kulkarni MV. 2004. Synthesis of spiro[indolo-1,5benzodiazepines] from 3-acetyl coumarins for use as possible antianxiety agents. $J$. Chem. Sci., 116: 265-270.

Meslin JC, Quiniou H. 1974. The synthesis of 6-oxo- (and 6-thioxo)-6H-1,3-thiazines and 5-aroylthiazoles from thioaroyl $\mathrm{N}, \mathrm{N}$ dimethylformamidines. Synthesis, 298300.

Meslin JC, Quiniou H. 1975. Enchainements hétéroatomiques et leurs produits de cyclisation. N,N-diméthyl N'thioaroylformamidines, aroyl-5-yhiazoles et 6H-thiazine-1,3-ones (thiones)-6substitués. Tetrahedron, 31: 3055-3058.

Miller FD, Monto AS, DeLong DC, Exelby A, Bryan ER, Srivastava S. 1985. Controlled trial of enviroxime against natural rhinovirus infections in a community. Antimicrob. Agents Chemother., 27: 102106.

Patel K, Singh A. 2009. Synthesis, Characterization and Chelating Properties of Benzimidazol-Salicylic Acid Combined Molecule. E-Journal of Chemistry, 6(1): 281-288.

Porcari AR, Devivar RV, Kucera LS, Drach JC, Townsend LB. 1998. Design, synthesis, and antiviral evaluations of 1(substituted benzyl)-2-substituted-5,6dichlorobenzimidazoles as nonnucleoside analogues of 2,5,6-trichloro-1-((-dribofuranosyl)-benzimidazole. J. Med. Chem., 41: 1252-1262.

Richter JE. 1997. Long-term management of gastroesophageal reflux disease and its 
complications. Am. J. Gastroenterol., 92: 30-34.

Sanghetti JN, Kokare ND, Shinde DB. 2007. Sulfanic acid catalyzed solvent-free synthesis of 1,5-Benzodiazepine derivatives. Chinese Chem. Lett., 18: 1305-1308.

Sissouma D, Touré SA, Adjou A, Baba G, Téa GC, N'guessan YT. 2004. Reactivity of amidinium salts: application in the synthesis of thiosubstituted benzimidazoles. J. Soc. Ouest-Afr. Chim., 018: 1-19.

Swayze EE, Peiris SM, Kucera LS, White EL, Wise DS, Drach JC, Townsend LB. 1993. Synthesis of 1-(2-aminopropyl) benzimidazoles, structurally related to the TIBO derivative $\mathrm{R} 82$ with activity against human immunodeficiency virus, Bioorg. Med. Chem. Lett., 3: 543-546.

Téa CG, Pradère JP, Villieras J. 1983. $6 H$ Thiazines-1,3 substituées en position 2 par un groupement labile, électrophiles ambidents. Tetrahedron Lett. 24: 37133716.

Téa CG, Pradère JP, Quiniou H. 1985a. DielsAlder and retro Diels-Alder reactions from $N$ '-thioacylformamidines to thioamide vinylogs. J. Org. Chem., 50: 1545-1547.
Téa CG, Pradère JP, Quiniou H, Toupet L. 1985b. The preparation and rearrangement of 5-acyl-2-phenyl-4-substituted 6H-1,3thiazines. X-ray molecular structure of 3ethoxycarbonyl-4-(3-oxobutylthio)-5phenylpyrrole. J. Chem. Soc. Perkin Trans I: 1875-1878.

Téa CG, Pradère JP, Quiniou H. 1986a. Thermolysis of substituted 4H-1,3Thiazines. Synthetic Commun. 16: 79-83.

Téa CG, Chehna M, Pradère JP, Duguay G, Toupet L. 1986b. Thia-1-aza-3-butadiènes substitutes : Action du cétène et derives. Phosphorus and Sulfur, 27 : 327-339.

Ugi I, Aigner H, Glashsl G, Touré SA, zychlinsky HV. 1986. News reagents and methods for the synthesis of peptides $\beta$ lactams and oligonucleotides, natural product chemistry ATTA-UR RAHMAH SPRINGER-VERLAG BERLINHEIDELBERG, 457-484.

Victor F, Brown TJ, Campanale K, Heinz BA, Shirpley LA, Su SK, Tang J, Vance LM, Spitzer W 1997. Synthesis, antiviral activity and biological properties of vinylacetylene analogs of enviroxime. $J$. Med. Chem., 40: 1511-1518. 